\title{
Table ronde sur les fractures du rachis dorsal et lombaire
}

\author{
Cl. Schwartz ${ }^{1}$ et J.P. Steib ${ }^{2}$ \\ ${ }^{1}$ Service d'Orthopédie-Traumatologie, Centre Hospitalier Louis-Pasteur, F-68021 Colmar Cedex \\ ${ }^{2}$ Hôpital Chirurgical Orthopédique Stéphanie, F-67026 Strasbourg Cedex
}

Le bureau du GECO nous a confié cette année un sujet de traumatologie : « les fractures du rachis dorsal et lombaire ».

Ces fractures nous concernent tous, versés ou non dans la pathologie rachidienne. Chacun d'entre nous a été amené un jour à prendre en charge de tels blessés. Nous en tiendrons pour preuve le fait d'avoir pu rassembler 317 fractures, toutes traitées par les membres du GECO ou dans leur service. Il semblait donc important de faire le bilan de ce que nous avons fait ces dix dernières années et savoir si nous avons raison d'opérer de plus en plus ces fractures (deux fois plus en moins de 10 ans !).

Le sujet recouvre donc toutes les fractures de D1 à L5, toutefois plus de $50 \%$ se situent en D12, L1 et L2. Nous avons limité le sujet aux fractures fraîches traitées de première main par les membres du GECO. Mais il s'agit de fractures de tout-venant sans sélection préalable tenant à l'âge, à la gravité ou au type de traitement.

Nous n'avons voulu ni transformer cette Table ronde en symposium informatique et statistique distillant l'ennui, ni fomenter une guerre de religion entre ultra-spécialistes au risque de nuire à la compréhension du sujet. Il nous fallait gagner l'intérêt général et rendre cette Table ronde accessible à tous, sans lasser ceux qui connaissent bien le sujet pour le pratiquer tous les jours. Leur contribution est fondamentale car les choses évoluent très vite en la matière.

Dans un premier temps, nous allons nous rafraîchir la mémoire en nous rappelant l'anatomie, la biomécanique et la radiologie. Nous avons adopté la classification de Francis Denis qui est actuellement la plus diffusée et nous semble la plus logique. Celle-ci nous permettra d'utiliser un langage commun tirant un trait d'union entre les différents services. Puis, dans cette première partie toujours, après avoir revu la sémiologie neurologique de ces fractures, nous aborderons le traitement orthopédique et fonctionnel. C'est en fin de séance que nous commencerons l'analyse de la série du GECO.
L'étude statistique a été sérieuse et performante grâce au travail de J.M. Balliet. N'ont été retenus que les dossiers complets disposant d'un recul supérieur à 6 mois. Ainsi un grand nombre de dossiers a été refusé lors de cette analyse.

La deuxième partie de la Table ronde s'intéresse au traitement chirurgical, qu'il soit postérieur par matériel de Roy-Camille, Cotrel-Dubousset ou de Hartshill, ou qu'il soit antérieur, voire double. C'est là que nos invités nous feront part de leur grande expérience en la matière. Enfin, après avoir pris connaissance des traitements appliqués dans la série du GECO, nous nous intéresserons aux résultats de cette série en les interprétant avec beaucoup de prudence car, en dehors de la signification statistique, il fallait remettre les informations dans leur contexte pour ne pas faire mentir les chiffres. C'est alors que nous saurons ce que l'on peut retenir de notre expérience commune et surtout les conclusions qu'on peut en tirer pour l'avenir. 